\title{
The Role of Allergy Therapy in Chronic Rhinosinusitis: A Systematic Review
}

\author{
Jorge I. Contreras • Berrylin J. Ferguson • \\ Eric W. Wang $\cdot$ Stella Lee
}

Published online: 18 December 2012

(c) Springer Science+Business Media New York 2012

\begin{abstract}
The role of allergy in chronic rhinosinusitis (CRS) remains controversial. The objectives of the review were to determine if atopy plays a role in CRS, and to determine if allergy treatment impacts patient outcome. We screened 1,755 articles, resulting in 37 studies, of which 7 were prospective. No randomized controlled trials examining the benefit of immunotherapy in CRS were found in the literature. The study populations for the majority of the studies available were highly selected, evaluating patients failing medical therapy and often requiring endoscopic sinus surgery (ESS). Evidence from these studies suggests a high prevalence of atopy in CRS patients with a possible role of food allergy in CRS pathophysiology. Five of the 7 cohort studies in this review did not show a correlation between atopy and post-surgical outcome in CRS patients requiring ESS.
\end{abstract}

Keywords Atopy · Allergic rhinitis · Chronic rhinosinusitis $\cdot$ Nasal polyposis $\cdot$ Immunotherapy $\cdot$ Asthma

\footnotetext{
J. I. Contreras · B. J. Ferguson · E. W. Wang · S. Lee ( $₫)$

Division of Sinonasal Disorders and Allergy,

University of Pittsburgh Medical Center, Mercy Building D,

Suite 2100, 1400 Locust St, Pittsburgh 15219, PA, USA

e-mail: lees6@upmc.edu

J. I. Contreras

e-mail: contrerasji@upmc.edu

B. J. Ferguson

e-mail: fergusonbj@upmc.edu

E. W. Wang

e-mail: wangew@upmc.edu
}

\section{Introduction}

Chronic rhinosinusitis (CRS) is an inflammatory disorder without a currently known etiology. CRS is heterogeneous with a spectrum of phenotypes and is considered multifactorial. Allergic rhinitis (AR) has been linked to CRS pathology not only because the two disorders coexist but are also characterized by the common denominator of inflammation. The correlation between AR and CRS is controversial and the data remains mixed. Increasingly, CRS is recognized as an inflammatory disorder with associated innate and adaptive immune dysfunction. Recent literature suggesting a local IgE hypersensitivity in the patient with negative allergy testing provides insight into the complexity of the disorder and may explain response to anti-IgE therapy in "non-allergic patients" $[1 \bullet \bullet]$. In a previous publication therapeutic strategies for AR in controlling inflammation were proposed to also be beneficial in CRS patients especially in patients with both disorders [2•]. While the pathophysiology of CRS is certainly diverse and frequently elusive, symptom-specific therapy and avoidance in those patients with concomitant allergic disease has been proposed to potentially ameliorate potential drivers of inflammation. This systematic review examines the evidence regarding the role of atopy in CRS severity and treatment outcome.

\section{Definitions and Epidemiology of Atopy}

The definition of atopy varies in the literature and sometimes used interchangeably with allergy. In general, most would agree that atopy is a tendency to produce $\operatorname{IgE}$ to allergens and is a characteristic phenotype of diseases such as $\mathrm{AR}$ or as a disease modifier. The prevalence of atopy in 


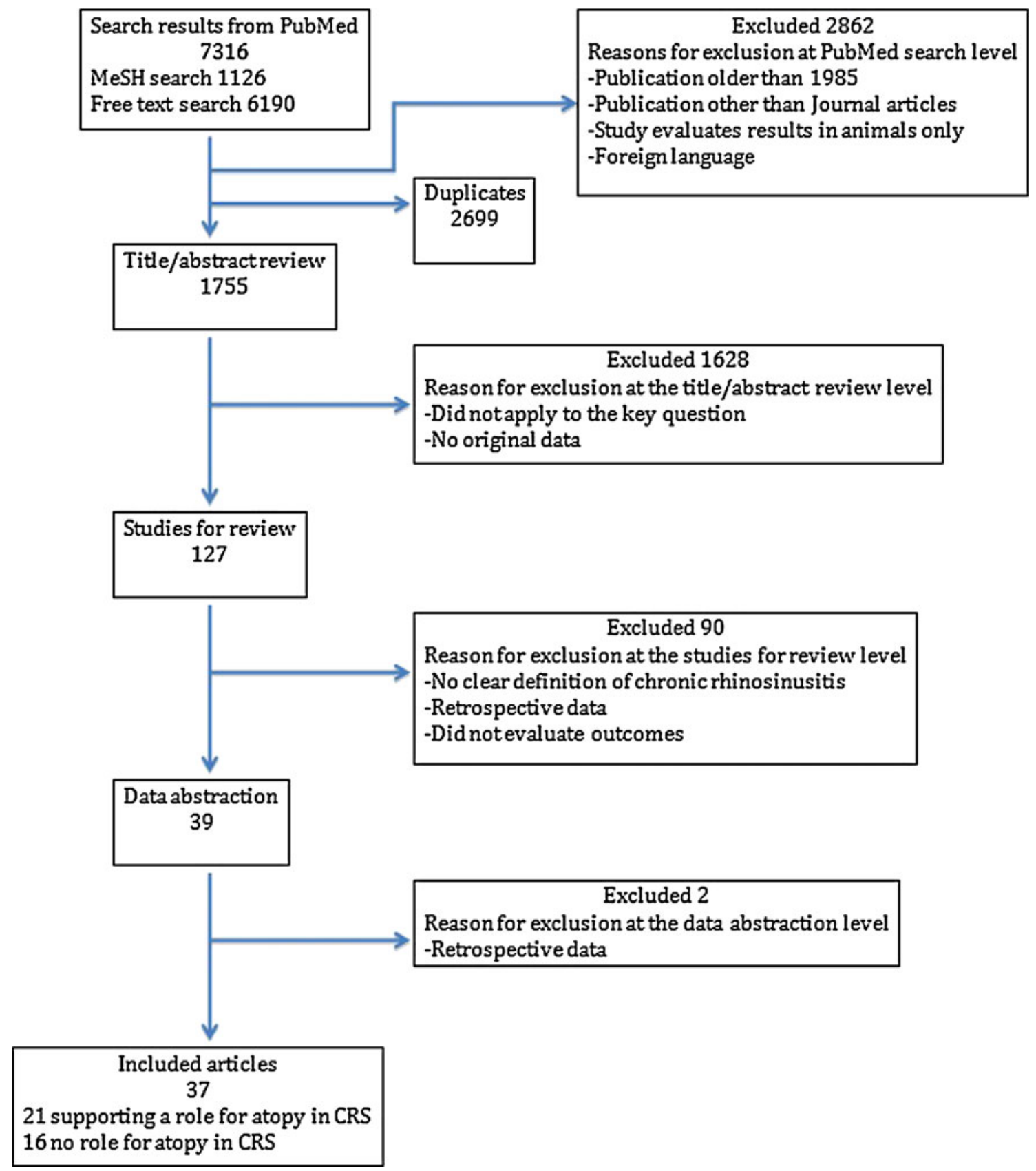

Fig. 1 Literature search flow

the general population is increasing. In the U.S. the prevalence of atopy based on the National Health and Nutrition Examination Survey 2005-2006 was $43.7 \%$, an increase of 2-5.5 times from several decades earlier [3•,4]. A recent $\mathrm{GA}^{2} \mathrm{LEN}$ survey showed that $56.7 \%$ of those reporting symptoms that fulfilled the $\mathrm{EP}^{3} \mathrm{OS}$ criteria for CRS also reported symptoms of allergy [5•]. The significance of these findings in the context of clinical relevance, i.e., symptom severity, quality of life outcomes, and treatment implications, however, is unclear.

\section{Systematic Review of Atopy and CRS}

The objectives of this review were to determine whether an association exists between atopy and chronic rhinosinusitis, as well as to determine if there is an impact of allergy treatment for patients with chronic rhinosinusitis. Prospective studies and controlled trials published in the English language evaluating adults or children with CRS with or without nasal polyps (NPs) in the setting of atopy (inhalant and food allergies) were included. Retrospective 
studies and reviews were excluded. Figure 1 shows a schematic of the literature search and selection.

\section{Outcome Measures}

Outcome measures including symptom scores, radiographic severity, incidence of patients requiring surgery, and surgical outcome were analyzed. Prevalence of atopy in CRS and the prevalence of aspirin-exacerbated respiratory disease (AERD) in comparison to control populations, if available, were also evaluated.

Search Methods for Identification and Selection of Studies

A literature review was conducted by two authors (JIC and SL) using PubMed for articles from 1985 through September 2012. A Medical Subject Headings search using the subject headings "rhinitis, allergic, seasonal" or "rhinitis, allergic, perennial" or "food hypersensitivity" and "sinusitis" or "nasal polyps" yielded 426 abstracts for review. An additional PubMed free text search using the terms "allergy," "chronic sinusitis," "rhinosinusitis," "nasal polyps," "allergic rhinitis," "atopy," "immunotherapy," and "food allergy" was conducted. This search strategy yielded an additional 1,329 abstracts, for review for a total of 1,755 abstracts. Reference lists from identified publications were scanned to identify further trials, which were missed by the initial search criteria, yielding an additional two abstracts. Quality of the studies was assessed, inclusion criteria applied independently, and any difference in opinion about which studies to include in the review was resolved by discussion and consensus. Thirty-seven studies met the inclusion criteria.

\section{Data Collection and Analysis}

A standardized data extraction form was utilized and each article underwent duplicate review. Differences in opinion were resolved by discussion and consensus.

A total of 1,755 papers were identified, and after appropriate exclusion 37 papers were included in the review. No randomized controlled studies were available in the literature. Prospective and controlled studies were included and all retrospective studies and reviews excluded. Twenty-one articles supported a role of atopy in CRS while 16 papers showed no association.

\section{Prevalence of Atopy in CRS Patients}

The prevalence of atopy reported in patients with CRS and/ or nasal polyposis varied widely, 16-85\% for inhalant sensitivities and $12-81 \%$ for food sensitivities. The study populations were highly selected with many of the studies enrolling patients who had failed medical therapy or already undergone endoscopic sinus surgery. Dust mite and mold sensitivities as well as food sensitization were more common in controlled studies that suggested a role for atopy in CRS. It was found that prevalence measurements varied based on the particular time point at which a study was conducted as well as the population studied. The majority of studies, however, did not consistently provide prevalence measurements in the context of normative data for that time point and population. The studies included in this review also showed variability in allergy testing methods including skin prick, intradermal, and measurements of serum-specific IgE as well variability in the diagnosis of CRS (Table 1).

\section{Studies Supporting a Role for Atopy in CRS Patients}

A total of 21 studies showed a role for atopy in patients with CRS or nasal polyposis, with 1 prospective cohort study (evidence level 2b), 16 case-control studies (evidence level 3b), and 4 case-series (evidence level 4). The one cohort study evaluated the role of allergy treatment including immunotherapy on CRS outcome in pediatric patients and found that medical therapy before surgery with topical nasal steroid sprays, oral antihistamines, and immunotherapy (in $25 \%$ of patients) improved the surgical success rate at a 1 year follow up from 64 to $84 \%$ [6]. The authors proposed that treatment of allergy before surgery could help improve outcome. In this study, however, the authors describe that all patients underwent allergy evaluation but did not describe their specific method of allergy testing.

In a recent study, Lin et al. [7•] found a higher rate of surgery in CRS patients with AR, as well as increased symptom severity and likelihood of medication use. There was a prevalence of $65 \%$ of atopy in the CRS patients compared to $18 \%$ in the control group. Other studies have also showed that the presence of allergy increased symptom severity [8-11]. These studies utilized validated symptom scores including the SF-36, rhinosinusitis disability index (RSDI), and the sinonasal outcome test (SNOT-20).

The majority of studies found an association with perennial allergen sensitivities and CRS or nasal polyposis, specifically dust mite [10, 12•,13-15] and mold [16, 17]. In a Spanish study evaluating 121 patients with nasal polyps, $63 \%$ had positive skin prick testing compared to $31 \%$ of controls. Dermatophagoides pteronyssinus was the most common allergen eliciting a response [13]. The authors suggested a possible IgE-mediated hypersensitivity 


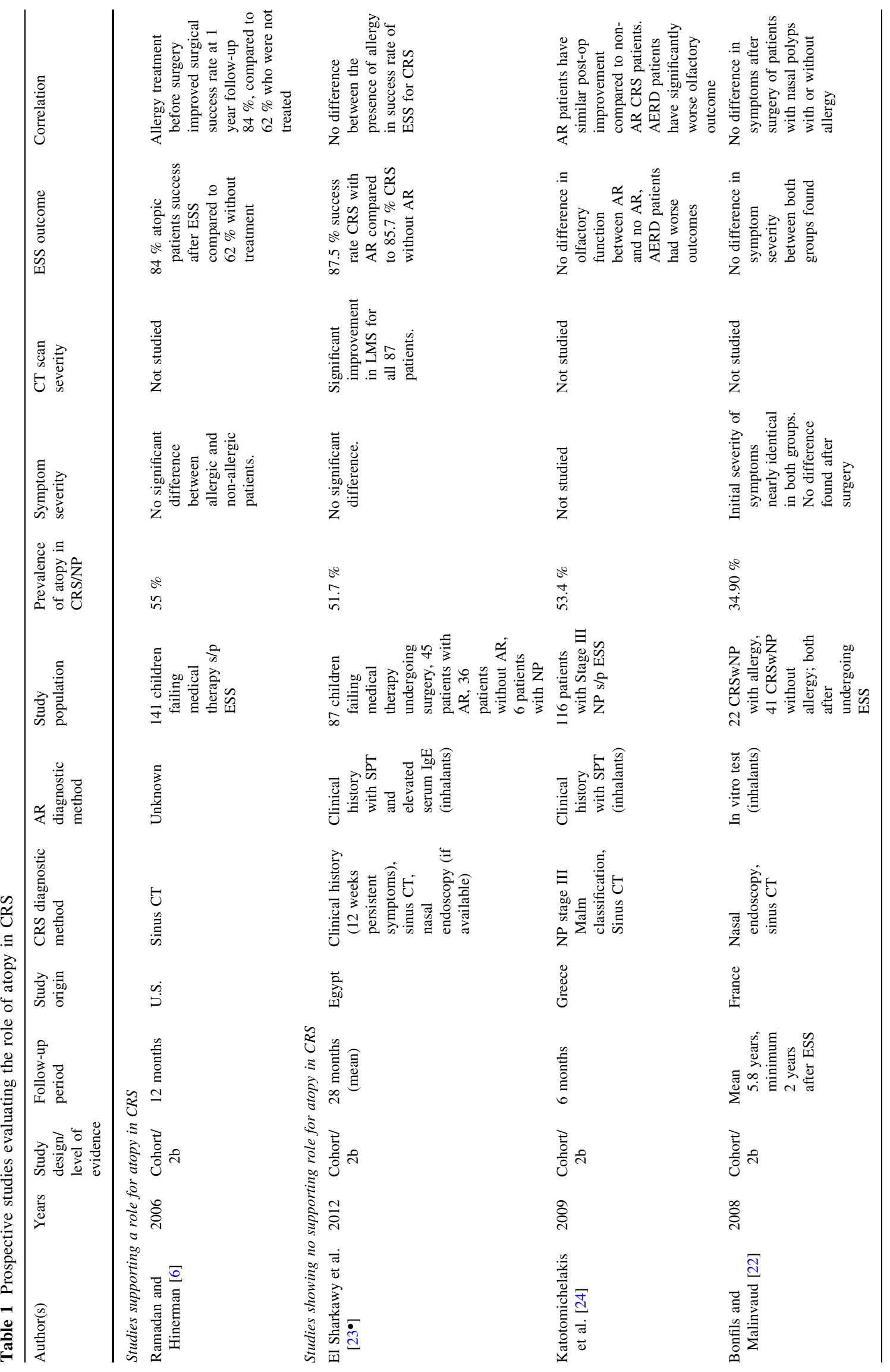


Curr Otorhinolaryngol Rep (2013) 1:33-44

37

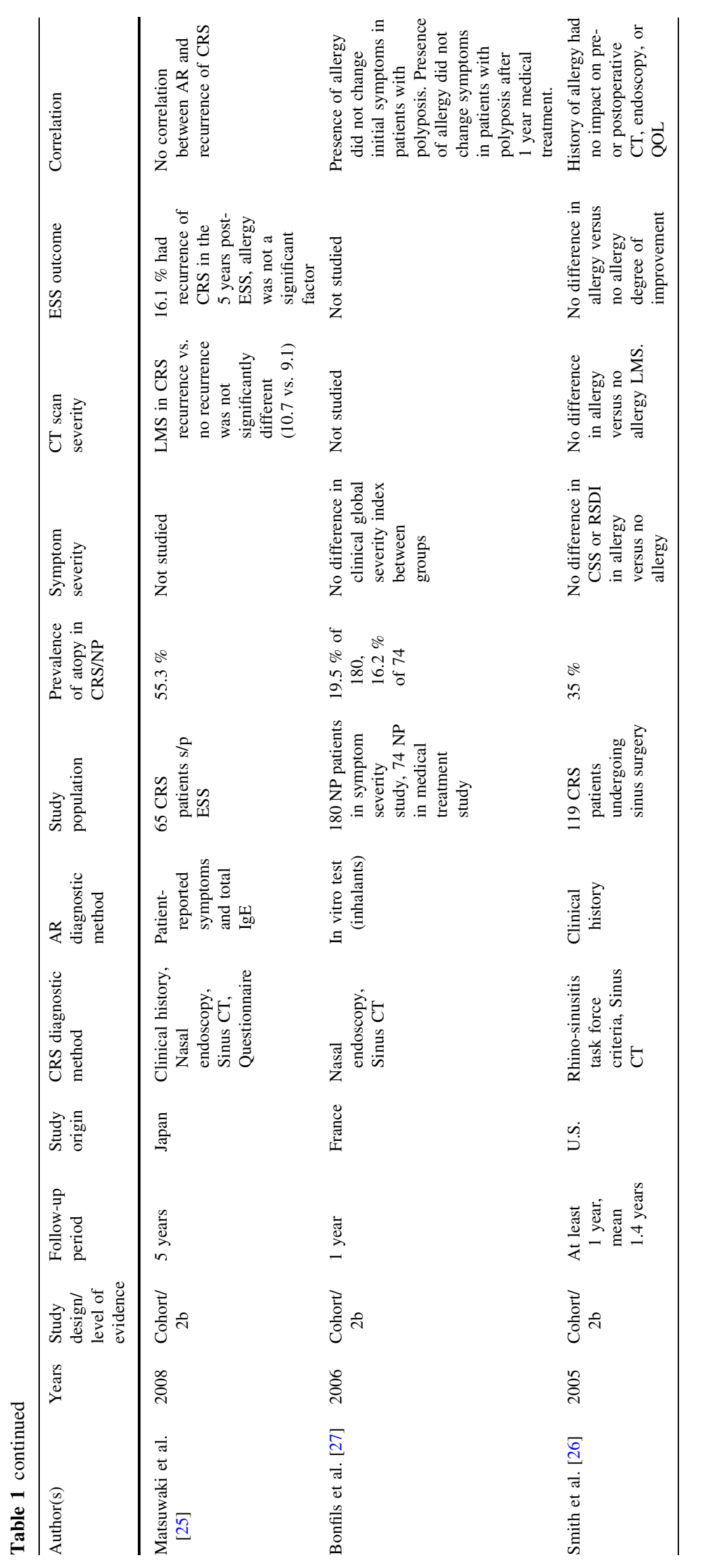

Springer 
in the development of polyps. A study of CRS patients who failed medical therapy, found that $82 \%$ were positive on skin prick testing with higher rates of asthma, and multiple allergen sensitivity especially to dust mite and ragweed in CRS with NP patients compared to controls [12•].

A correlation has been suggested with NPs and food allergy and four case-control studies were included in our review. A higher rate of milk allergy was seen in CRS with NP compared to controls, whereas no difference was seen for wheat sensitization [18•]. Patients undergoing surgery for NPs were also more likely to have positive food SPT compared to controls (70 vs. $34 \%$ ). The prevalence of positive inhalant SPT, however, was not significantly different between patients with NP compared to controls (43 vs. $48 \%$ ) [19]. A Turkish study showed a higher rate of food sensitization in NP patients in comparison to controls (73.5 vs. $15 \%)$ with the most common reactions to onion, oat, grapefruit, plum, buckwheat, and apple [20]. Other food reactions included wheat, potato, and tomato in a similar study from Singapore, which found a significantly higher prevalence of food sensitivity in NP patients compared to controls (81 vs. $11 \%$ ) [21]. The authors in this study concluded that the presence of salicylates in food may not contribute to the pathogenesis of NPs since only 1 patient reacting to the salicylate-containing foods tested, tomato and/ or pea, was found.

IDT to food allergens has been shown to be more sensitive than SPT for detecting atopy, perhaps due to a bigger allergen dose to register a response [22]. The pathophysiology of food allergy reactions remains unclear with type II, III, and IV delayed hypersensitivity reactions also proposed to contribute to chronic inflammation. It is interesting to note that the majority of study patients did not report a history of food hypersensitivity [21, 22, 23•, 24].

None of the studies evaluated whether exposure to the foods found positive on testing resulted in worsening symptoms, CT scan scores, post-surgical outcome, or whether elimination diets led to resolution of inflammation.

\section{Studies Showing no Role of Atopy in CRS Patients}

A total of 16 studies showed no association between atopy and CRS or nasal polyps, including 6 cohort studies, 2 casecontrol studies, and 8 case-series (Table 2). Five of the prospective studies found no differences between the presence of atopy, post-operative improvement, and symptoms after endoscopic sinus surgery [22, 23•, 24-26]. In a recent study by El Sharkawy et al. there was no difference in surgical success rate after a mean follow-up of 28 months in pediatric patients with or without allergy. The definition of success was based on improvement in sinus CT 8 weeks post-operatively, nasal exam, and an un-validated symptom survey. Olfactory outcome measured by Sniffin' Sticks in a study of 116 patients with NPs found no difference between allergic and non-allergic groups, although patients with AERD had a worse olfactory outcome [24]. Similarly, a French study evaluating symptom severity after ESS reported no difference after a minimum 2 year follow-up (mean 5.8 years) between patients with and without atopy [22]. This study used an un-validated symptom severity survey measuring degree of nasal obstruction, rhinorrhea, and loss of smell. Rather than a history of allergy or total $\mathrm{IgE}$, local eosinophilia was the most predictive parameter for recurrent disease in a 5 year follow-up study of 65 patients with CRS who had undergone ESS. In this study, however, antigen-specific IgE was not measured and a history of allergy was obtained via an unvalidated questionnaire. Atopy also did not appear to play a role in CRS patients when examining medication usage including topical nasal steroid sprays, irrigations, and oral corticosteroids over a period of 1 year [27]. In this study, nasal function was evaluated on a 3-point scale evaluating nasal obstruction, anterior/posterior rhinorrhea, facial pain, and loss of smell.

Of the 17 studies that evaluated symptom severity, 10 studies reported no difference in symptom scores between atopic and non-atopic groups $[6,13,22,23 \bullet, 26,27,28 \bullet$, 29-31, 32•]. Only 2 of the 10 studies, however, used a validated symptom survey (RSDI, Chronic Sinusitis Survey, or Visual Analogue Scale) with the remaining studies utilizing un-validated symptom surveys, a clinical history of allergy or self-reported symptoms to determine allergic symptoms rather than an objective measure of atopy $[25,26]$.

Ten of the 12 studies examining CT scan severity showed that the presence of atopy did not appear to significantly influence Lund-Mackay scores (LMS) [8, 12•, $25,26,29,30,32 \cdot 33-35]$. Although no difference between endoscopic or LMS of patients with NPs were seen there were higher levels of IL-4, IL-5, IL-6, and eosinophils in atopic NP patients compared to non-atopic patients [32•]. In a case series, Robinson et al. [11] showed that there was no significant difference in rate of revision surgery in atopic compared to non-atopic patients (38 vs. $45.2 \%$ ), but there was a modest increase in mean LMS in atopic patients. Another case series showed an association between CT stage and mean end point with increased skin sensitivity correlating with more severe disease on CT [9].

\section{Atopy, CRS, and AERD}

Since patients with AERD have increased severity of disease with high recurrence of polyps, the role of atopy in these studies was evaluated to determine potential confounding of data. Eleven of the studies reported the prevalence of AERD [20, 24, 25, 27, 29, 31, 35, 37-40]. The 


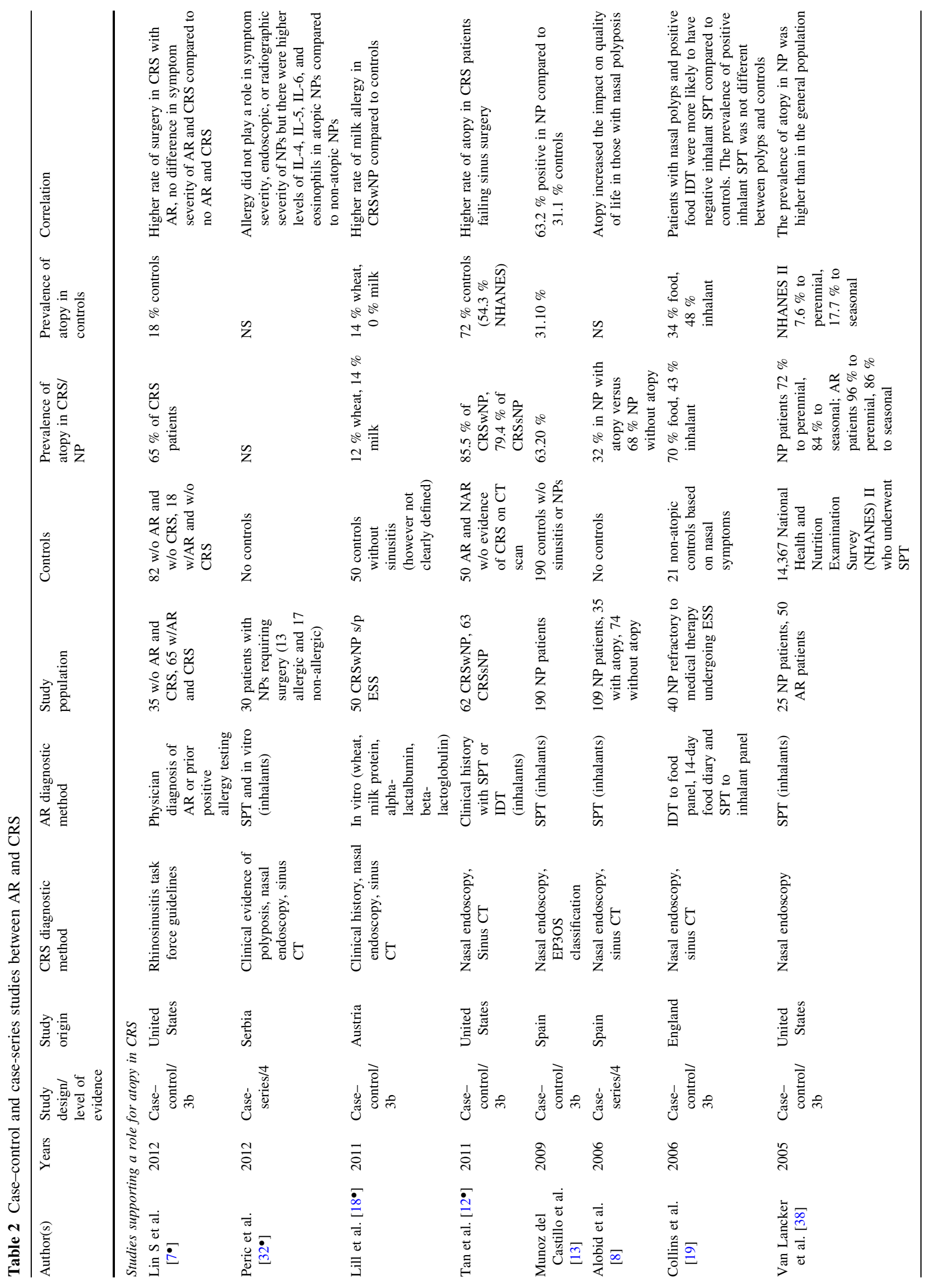




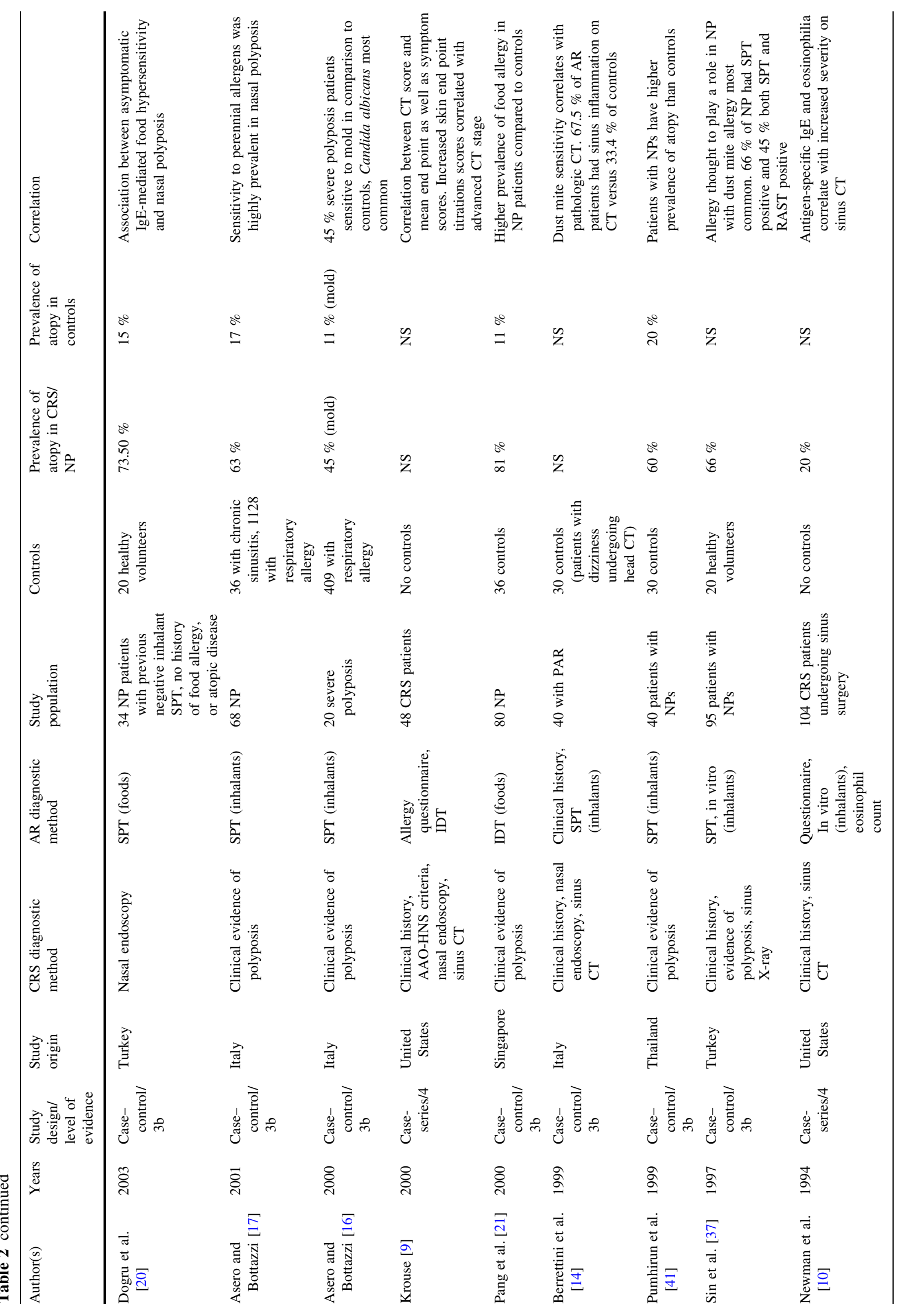




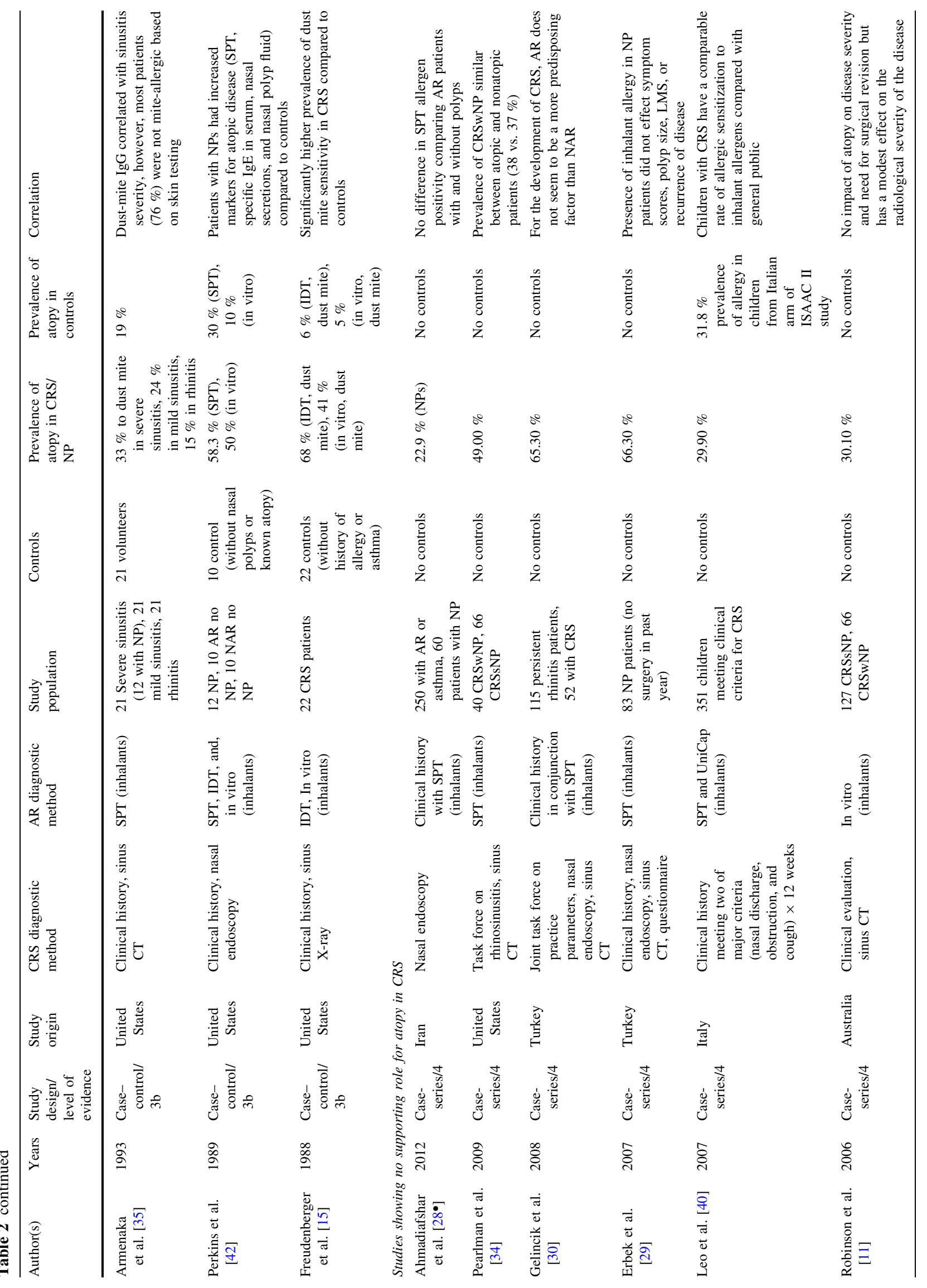




\begin{tabular}{|c|c|c|c|c|}
\hline & 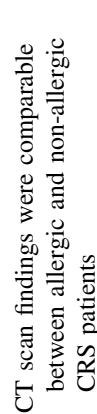 & 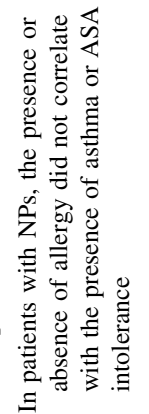 & 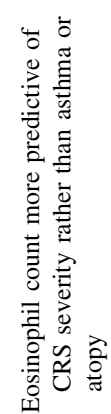 & 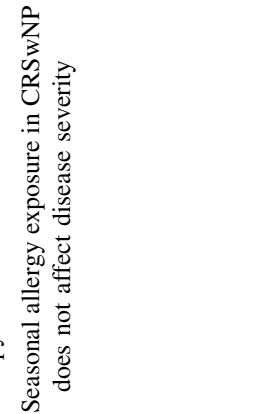 \\
\hline 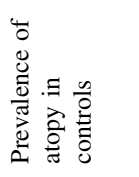 & $\begin{array}{l}\frac{n}{0} \\
\bar{\theta} \\
0 \\
0 \\
\check{z}\end{array}$ & $\therefore$ & 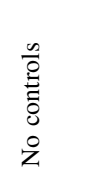 & 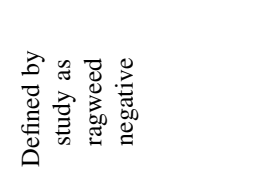 \\
\hline 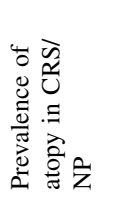 & $\begin{array}{l}\circ \\
\infty \\
i \\
0\end{array}$ & & $\begin{array}{l}\stackrel{9}{q} \\
\text { g }\end{array}$ & 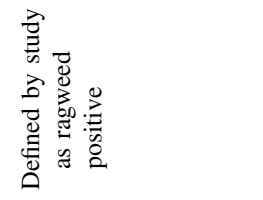 \\
\hline & $\begin{array}{l}n \\
0 \\
0 \\
0 \\
0 \\
0 \\
0\end{array}$ & 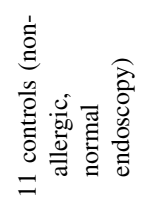 & $\begin{array}{l}n \\
0 \\
0 \\
\vdots \\
0 \\
0 \\
0 \\
z\end{array}$ & 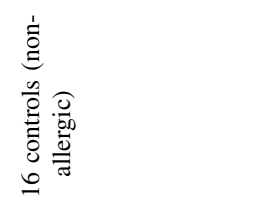 \\
\hline 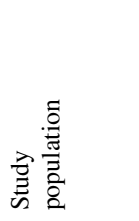 & $\begin{array}{l}\tilde{U} \\
\text { हे } \\
\text { }\end{array}$ & 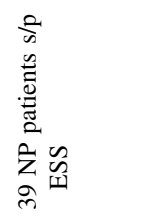 & 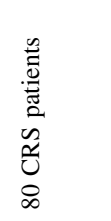 & 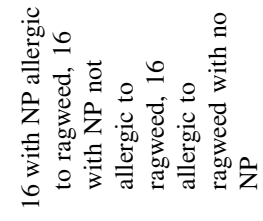 \\
\hline 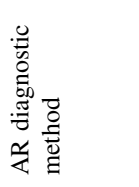 & 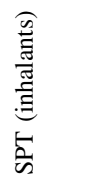 & 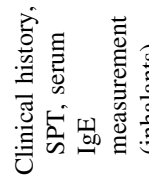 & 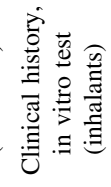 & 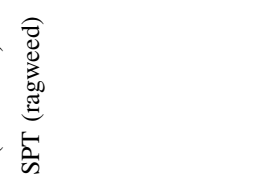 \\
\hline 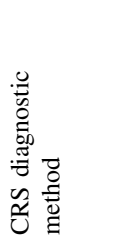 & 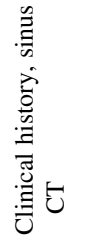 & 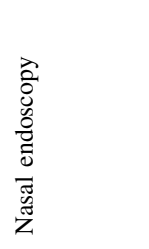 & 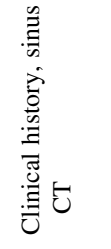 & 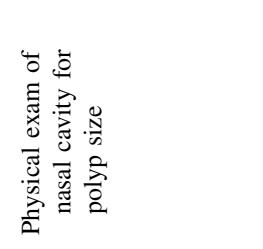 \\
\hline 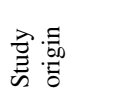 & 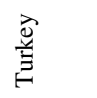 & $\begin{array}{l}\overline{\mathbb{N}} \\
\stackrel{\bar{n}}{n}\end{array}$ & 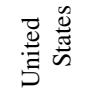 & $\begin{array}{l}\text { 烝 } \\
\text { Uू }\end{array}$ \\
\hline 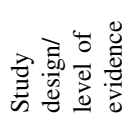 & 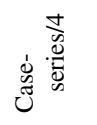 & 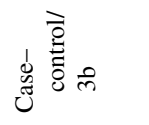 & 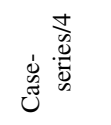 & 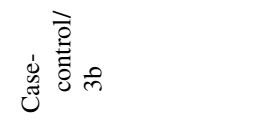 \\
\hline 营 & ષ્ণ & $\overline{\check{d}}$ & $\hat{\sigma}$ & $\stackrel{+}{\sigma}$ \\
\hline 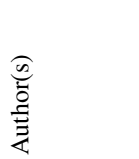 & 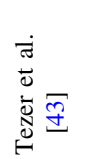 & 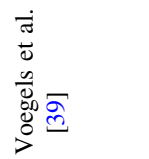 & 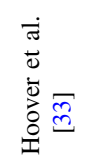 & 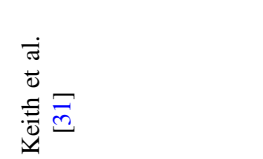 \\
\hline
\end{tabular}


prevalence was reported between 2 and $21.9 \%$ and did not appear to differ between patients with atopy, non-allergic rhinitis, nasal polyposis, and controls. Although patients with AERD demonstrate increased rates of recurrence of disease and severity of symptoms, atopy did not appear to play a significant role in these patients.

\section{Allergy Testing and Immunotherapy in CRS Patients}

Based on current evidence and guidelines, allergy testing in the management of CRS patients is optional [36•]. The evidence in this systematic review suggests that allergy and CRS commonly co-exist and therefore allergy testing and immunotherapy, if appropriate, is warranted for the treatment of AR. Whether this treatment impacts CRS outcome is unclear. No randomized controlled trials investigating the role of immunotherapy in CRS treatment have been performed. With the absence of evidence and heterogeneity of data, no formal recommendation can be made regarding immunotherapy in CRS patients.

Symptoms of allergy and rhinosinusitis often overlap and it is often difficult to distinguish one from the other clinically. In our practice, allergy evaluation and treatment is recommended for all CRS patients who have undergone an initial trial of medical therapy without success, and immunotherapy is recommended for patients with inadequate relief with environmental controls in conjunction with medical therapy or with symptoms for at least 6 months of the year. Patient who also have a clinical history of food intolerance or hypersensitivity are also tested for food allergy and counseling is provided on elimination or avoidance if appropriate.

\section{Conclusion}

The evidence suggesting a role for atopy in CRS is limited, although it appears that the two disorders commonly co-exist and food allergy may play a role. Only one prospective study evaluating the potential role for immunotherapy in patients with AR and CRS met criteria for inclusion suggesting that allergy immunotherapy helps improve post-surgical outcome. The studies evaluated in this review also suggest that atopy does not play a significant role in CT scan severity or post-surgical outcome in patients with CRS or nasal polyposis. Future study is needed to determine the etiology for the higher prevalence of food allergy in patients with CRS and whether allergy immunotherapy or avoidance can improve patient outcome.

Disclosure No potential conflicts of interest relevant to this article were reported.

\section{References}

Papers of particular interest, published recently, have been highlighted as:

- Of importance

•• Of major importance

1. • Gevaert P, Calus L, Van Zele T et al. Omalizumab is effective in allergic and nonallergic patients with nasal polyps and asthma. J Allergy Clin Immunol. 2012. doi:10.1016/j.jaci.2012.07.047. A randomized, double-blind, placebo-controlled trial of allergic and non-allergic patients with nasal polyps treated with omalizumab showed improvement in polyp scores, nasal/asthma scores, and quality of life irrespective of allergy.

2. - Lee S, Kundaria S, Ferguson BJ. Practical clinical management strategies for the allergic patient with chronic rhinosinusitis. Curr Opin Otolaryngol Head Neck Surg 2012;20:179-187. This review provides an algorithm for the treatment of patients with allergic rhinitis and CRS.

3. - Salo PM, Calatroni A, Gergen PJ et al. Allergy-related outcomes in relation to serum IgE: results from the National Health and Nutrition Examination Survey 2005-2006. J Allergy Clin Immunol. 2011;127:1226-1235 e1227. The most current iteration of NHANES, this study provides updated data on atopic prevalence in the U.S. as well as allergy-related outcomes.

4. Arbes SJ Jr, Gergen PJ, Elliott L, Zeldin DC. Prevalences of positive skin test responses to 10 common allergens in the US population: results from the third National Health and Nutrition Examination Survey. J Allergy Clin Immunol. 2005;116:377-83.

5. - Jarvis D, Newson R, Lotvall $\mathrm{J}$ et al. Asthma in adults and its association with chronic rhinosinusitis: The GA(2) LEN survey in Europe. Allergy 2012;67:91-98. This study examines the prevalence of asthma, allergic rhinitis, and its association with CRS across 12 countries.

6. Ramadan HH, Hinerman RA. Outcome of endoscopic sinus surgery in children with allergic rhinitis. Am J Rhinol. 2006;20: 438-40.

7. - Lin SY, Reh DD, Navas-Acien A. Allergic rhinitis, chronic rhinosinusitis, and symptom severity: a population-based study. Int Forum Allergy Rhinol. 2012;2:51-56. This community-based case-control study evaluates the relationship between AR, CRS, and impact on nasal symptoms.

8. Alobid I, Benitez P, Valero A, et al. The impact of atopy, sinus opacification, and nasal patency on quality of life in patients with severe nasal polyposis. Otolaryngol Head Neck Surg. 2006;134:609-12.

9. Krouse JH. Computed tomography stage, allergy testing, and quality of life in patients with sinusitis. Otolaryngol Head Neck. 2000;123:389-92.

10. Newman LJ, Platts-Mills TA, Phillips CD, et al. Chronic sinusitis. Relationship of computed tomographic findings to allergy, asthma, and eosinophilia. J Am Med Assoc. 1994;271:363-7.

11. Robinson S, Douglas R, Wormald PJ. The relationship between atopy and chronic rhinosinusitis. Am J Rhinol. 2006;20:625-8.

12. - Tan BK, Zirkle W, Chandra RK et al. Atopic profile of patients failing medical therapy for chronic rhinosinusitis. Int Forum Allergy Rhinol. 2011;1:88-94. This study evaluated the atopic status of patients undergoing sinus surgery for CRS refractory to medical therapy.

13. Munoz del Castillo F, Jurado-Ramos A, Fernandez-Conde BL, et al. Allergenic profile of nasal polyposis. J Investig Allergol Clin Immunol. 2009;19:110-6.

14. Berrettini S, Carabelli A, Sellari-Franceschini S, et al. Perennial allergic rhinitis and chronic sinusitis: correlation with rhinologic risk factors. Allergy. 1999;54:242-8. 
15. Freudenberger T, Grizzanti JN, Rosenstreich DL. Natural immunity to dust mites in patients with chronic rhinosinusitis. J Allergy Clin Immunol. 1988;82:855-62.

16. Asero R, Bottazzi G. Hypersensitivity to molds in patients with nasal polyposis: a clinical study. J Allergy Clin Immunol. 2000; 105:186-8.

17. Asero R, Bottazzi G. Nasal polyposis: a study of its association with airborne allergen hypersensitivity. Ann Allergy Asthma Immunol. 2001;86:283-5.

18. - Lill C, Loader B, Seemann R et al. Milk allergy is frequent in patients with chronic sinusitis and nasal polyposis. Am J Rhinol Allergy. 2011;25:e221-4. This study examined the relationship of wheat and milk allergies to chronic polypoid sinusitis and recurrent disease.

19. Collins MM, Loughran S, Davidson P, Wilson JA. Nasal polyposis: prevalence of positive food and inhalant skin tests. Otolaryngol Head Neck Surg. 2006;135:680-3.

20. Dogru H, Tuz M, Uygur K, et al. Asymptomatic IgE mediated food hypersensitivity in patients with nasal polyps. Asian Pac J Allergy Immunol. 2003;21:79-82.

21. Pang YT, Eskici O, Wilson JA. Nasal polyposis: role of subclinical delayed food hypersensitivity. Otolaryngol Head Neck Surg. 2000;122:298-301.

22. Bonfils P, Malinvaud D. Influence of allergy in patients with nasal polyposis after endoscopic sinus surgery. Acta Otolaryngol. 2008;128:186-92.

23. - El Sharkawy AA, Elmorsy SM, Eladl HM. Functional endoscopic sinus surgery in children: predictive factors of outcome. Eur Arch Otorhinolaryngol 2012;269:107-111. A prospective cohort study examining the role of atopy in pediatric patients undergoing sinus surgery, the results suggested no difference between atopic and non-atopic groups.

24. Katotomichelakis M, Riga M, Davris S, et al. Allergic rhinitis and aspirin-exacerbated respiratory disease as predictors of the olfactory outcome after endoscopic sinus surgery. Am J Rhinol Allergy. 2009;23:348-53.

25. Matsuwaki Y, Ookushi T, Asaka D, et al. Chronic rhinosinusitis: risk factors for the recurrence of chronic rhinosinusitis based on 5-year follow-up after endoscopic sinus surgery. Int Arch Allergy Immunol. 2008;146(Suppl 1):77-81.

26. Smith TL, Mendolia-Loffredo S, Loehrl TA, et al. Predictive factors and outcomes in endoscopic sinus surgery for chronic rhinosinusitis. Laryngoscope. 2005;115:2199-205.

27. Bonfils P, Avan P, Malinvaud D. Influence of allergy on the symptoms and treatment of nasal polyposis. Acta Otolaryngol. 2006;126:839-44.

28. - Ahmadiafshar A, Farjd HR, Moezzi F, Mousavinasab N. Nasal polyposis in patients with asthma and allergic rhinitis. J Laryngol Otol. 2012;126:780-783. A case series evaluating CRS patients with and without AR, no difference was found in SPT allergen positivity in the two groups.

29. Erbek SS, Erbek S, Topal O, Cakmak O. The role of allergy in the severity of nasal polyposis. Am J Rhinol. 2007;21:686-90.

30. Gelincik A, Buyukozturk S, Aslan I, et al. Allergic vs nonallergic rhinitis: which is more predisposing to chronic rhinosinusitis? Ann Allergy Asthma Immunol. 2008;101:18-22.

31. Keith PK, Conway M, Evans S, et al. Nasal polyps: effects of seasonal allergen exposure. J Allergy Clin Immunol. 1994;93: 567-74.

32. - Peric A, Vojvodic D, Vukomanovic-Durdevid B. Influence of allergy on clinical, immunological and histological characteristics of nasal polyposis. B-Ent 2012;8:25-32. A case series of 30 patients requiring endoscopic sinus surgery found that allergy does not modify symptom or radiographic severity.

33. Hoover GE, Newman LJ, Platts-Mills TA, et al. Chronic sinusitis: risk factors for extensive disease. J Allergy Clin Immunol. 1997; 100:185-91.

34. Pearlman AN, Chandra RK, Chang D, et al. Relationships between severity of chronic rhinosinusitis and nasal polyposis, asthma, and atopy. Am J Rhinol Allergy. 2009;23:145-8.

35. Armenaka MC, Grizzanti JN, Oriel B, Rosenstreich DL. Increased immune reactivity to house dust mites in adults with chronic rhinosinusitis. Clin Exp Allergy. 1993;23:669-77.

36. - Meltzer EO, Hamilos DL. Rhinosinusitis diagnosis and management for the clinician: a synopsis of recent consensus guidelines. Mayo Clin Proc. 2011;86:427-443. This review examined and compared the current recommendations from several evidence-based guidelines.

37. Sin A, Terzioğlu E, Kokuludağ A, et al. Allergy as an etiologic factor in nasal polyposis. J Investig Allergol Clin Immunol. 1997;7:234-7.

38. Van Lancker JA, Yarnold PA, Ditto AM, et al. Aeroallergen hypersensitivity: comparing patients with nasal polyps to those with allergic rhinitis. Allergy Asthma Proc. 2005;26:109-12.

39. Voegels RL, Santoro P, Butugan O, Formigoni LG. Nasal polyposis and allergy: is there a correlation? Am J Rhinol. 2001;15: 9-14.

40. Leo G, Piacentini E, Incorvaia $\mathrm{C}$, et al. Chronic rhinosinusitis and allergy. Pediatr Allergy Immunol. 2007;18(Suppl):19-21.

41. Pumhirun P, Limitlaohapanth C, Wasuwat P. Role of allergy in nasal polyps of Thai patients. Asian Pac J Allergy Immunol. 1999;17:13-5.

42. Perkins JA, Blakeslee DB, Andrade P. Nasal polyps: a manifestation of allergy? Otolaryngol Head Neck Surg. 1989;101:641-5.

43. Tezer MS, Tahamiler R, Canakcioglu S. Computed tomography findings in chronic rhinosinusitis patients with and without allergy. Asian Pac J Allergy Immunol. 2006;24:123-7. 